\title{
Importance of Intelligible Phonemes for Human Speaker Recognition in Different Channel Bandwidths
}

\author{
Laura Fernández Gallardo ${ }^{1,2}$, Sebastian Möller ${ }^{2,1}$, Michael Wagner ${ }^{1,2,3}$ \\ ${ }^{1}$ Faculty of ESTeM, University of Canberra, Australia \\ ${ }^{2}$ Quality and Usability Lab, Telekom Innovation Laboratories, TU Berlin, Germany \\ ${ }^{3}$ College of Engineering and Computer Science, Australian National University, Australia \\ (laura.fernandez-gallardo|sebastian.moeller) dtelekom.de, michael.wagnerecanberra.edu.au
}

\begin{abstract}
It is known that nasal consonants and vowels are more effective than other phonemes for human speaker recognition. However, the influence of channel transmissions on the speakerdiscriminative capabilities of phonemes has not yet been examined. Specifically, the speech bandwidth has a strong effect on the human speaker recognition performance and also on the speech intelligibility. The phonemes that permit more accurate human speaker recognition are determined in this study by means of a speaker verification auditory test, focusing on the differences in performance when the stimuli are presented to listeners in narrowband and in wideband. The speech intelligibility is also investigated via an intelligibility test employing the same speech stimuli. Finally, the possible relationship between phonemes offering better human speaker recognition and more intelligible phonemes in the transition to an enhanced bandwidth is discussed.
\end{abstract}

Index Terms: human speaker identification, intelligibility, channel bandwidth

\section{Introduction}

The task of human speaker recognition is performed in everyday situations when we associate a voice we hear to a voice heard before and by some means encoded in memory. Features or cues employed by humans to distinguish among speakers as well as causes for performance variability have been investigated in the last decades. This research benefits forensic speaker recognition, cochlear implants, and telephony, among other areas. Different levels of voice pitch, loudness, breathiness, or laryngealisation enable listeners to discriminate among known talkers [1,2]. Humans also rely on acoustic correlates of phoneme articulations to identify speakers [3]. These features appear to be distinctive of the individuals and are crucial as well for the task of word recognition.

The degree to which different speech sounds convey speaker-specific characteristics was investigated in $[4,5,6] \mathrm{em}$ ploying automatic speaker recognition techniques. It was found that vowels and nasals provide the best discrimination between speakers, that fricatives contribute to the speaker recognition performance to a lesser extent, and that stop sounds are less useful for this task [6]. Regarding human speaker identification, the importance of vowel sounds is commonly acknowledged. Front stressed vowels [7] and nasalized vowels [8] have been found to be particularly useful. The works in $[9,10]$ have evinced that nasal sounds facilitate higher human speaker recognition than other consonants. This is attributable to the fact that the resonance cavities shaping nasal sounds differ considerably among speakers [11].

It is assumed that human speaker recognition and speech intelligibility are closely interrelated, as both are performed from linguistic cues. An in-depth review of this is given in [12] from a psychological point of view. The human capability to recognize speech has often been evaluated with different intelligibility tests. For instance, the rhyme tests, typically used in the 1980s, have been criticised for providing results biased towards more frequent words in a language. This difficulty is overcome with the CLuster IDentification test (CLID), which involves monosyllabic words generated according to the phonotactic relations and constraints in a language [13].

Background noise is considered one of the main factors affecting speech intelligibility of logatomes (nonsense syllables in the form vowel-consonant-vowel (VCV) or CVC) and of CV syllables $[14,15,16]$. Other intelligibility tests have examined the effects of speech bandwidth. It was found in [17] that the acoustic cues in the high-frequency regions (above $4 \mathrm{kHz}$ ) were redundant with those in the mid-frequency regions $(0.8-4 \mathrm{kHz})$, employing CVC logatomes. The results in [18] indicated that the frequencies around $1,370 \mathrm{~Hz}$ and $2,500 \mathrm{~Hz}$ contributed to speech intelligibility (from sentences and from phonetically balanced words) more than other examined bands (up to 9,500 Hz). The intelligibility of VCV and CV segments decreased around $5 \%$ when comparing a headphone condition (clean channel) and a handset condition (telephone channel). The same conditions were also considered in [19], employing meaningful utterances. Only marginal effects of bandwidth reduction on initial and final consonant intelligibility were reported.

Despite the current rapid deployment of digital communications, the effects of different telephone transmissions on intelligibility have not been investigated much. Wideband channels (WB, 50-7,000 Hz) enable higher transmitted signal quality [20] and better human speaker identification performance [21] in comparison to the traditional and still predominant narrowband (NB, 300-3,400 Hz). It is commonly acknowledged that the low frequencies incorporated contribute to increased naturalness, presence, and comfort, whereas the high frequency extension facilitates fricative differentiation [22]. However, no formal intelligibility test that evinces this last fact is known to the authors.

The present work takes a close look into the correspondences between intelligible phonemes and phonemes enabling better speaker recognition when moving from NB to WB. In other words, it examines whether better phoneme detection enabled by speech of greater bandwidth leads to higher speaker recognition rates for certain phonemes or phoneme classes. 
To this end, both a human speaker recognition test (speaker verification auditory test) and an intelligibility test were conducted employing the same audio material transmitted through NB and through WB channels. In order to study the effects of only certain phonemes as isolated as possible, VCV logatomes were employed as stimuli. A relatively old investigation [23] compared word intelligibility and speaker recognition over two real military communication channels, with a low-pass filter at $3 \mathrm{kHz}$, and in laboratory conditions. It was speculated that the channel impairments affecting intelligibility did not always affect human speaker recognition. In contradistinction, this paper examines the effects of NB and WB channels, commonly used in telephony nowadays, and focusing on phoneme differences.

\section{Audio Preparation}

Logatomes were employed as stimuli in the form VCV in order to study the effects of varying the vowel or the consonant sound only. Although it would have been desirable to conduct auditory tests with meaningful well-known words, it was not possible to find or to create a dataset of monosyllabic words differing in only one phoneme and not including different consonants or different vowels in one sample.

The Oldenburg Logatome Corpus (OLLO) [16] contains logatomes suitable for this study and was recorded in soundinsulated audiometry rooms (reverberation time $\approx 0.25 \mathrm{~s}$ ) with a studio-quality condenser microphone. These data were originally intended for speech intelligibility studies under the effects of masking noise [16]. Because the speech data in this dataset is clean, unprocessed, and with a sample frequency of $16 \mathrm{kHz}$, it was possible to transmit the speech through NB and WB communication channels. The different types of distortions applied to the signals could then be controlled in this manner.

The consonants selected for this study were:

- The fricatives $/ \mathrm{f} /, / \mathrm{s} /, / \mathrm{v} /$, and $/ \mathrm{f} /$

- The nasals $/ \mathrm{m} /$ and $/ \mathrm{n} /$

- The stop sounds $/ \mathrm{b} /$ and $/ \mathrm{p} /$

These eight consonants were embedded in the syllables /afa/, /asa/, /ava/, /aja/, /ama/, /ana/, /aba/, and /apa/ (in German: affa, assa, awwa, ascha, amma, anna, abba, and appa), and the stop consonant $/ \mathrm{p} /$ was also embedded in the four syllables /عpo/, /rpi/, /opo/, and / vpu/ (in German: eppe, ippi, oppo, and uppu). These twelve logatomes were selected from the OLLO set of ten German speakers (five males and five females) of standard High German dialect.

The transmission channels studied were a NB channel with the codec AMR-NB operating at $4.75 \mathrm{kbit} / \mathrm{s}$ and a WB channel with the codec G.722 at $64 \mathrm{kbit} / \mathrm{s}$. The process for speech distortion was applied as follows. First, the speech was level-equalised $26 \mathrm{~dB}$ below the overload of the digital system ( $-26 \mathrm{dBov})$, a characteristic level of telephone channels, using the voltmeter algorithm of the International Telecommunication Union (ITU-T) Recommendation P.56. For the NB channel, the signal was downsampled to $8 \mathrm{kHz}$ via an anti-aliasing low-pass FIR filter and then band-passed according to the ITU-T Recommendation G.712 standard implementation. This filter has a flat band-pass response over $300-3,400 \mathrm{~Hz}$ approximately. Then, the AMR-NB codec was simulated by employing the tools provided by the European Telecommunications Standards Institute (ETSI), specified in ETSI EN 301 704. Finally, the codeddecoded speech was again level-equalised to $-26 \mathrm{dBov}$.
For the WB channel, the original signals, sampled at $16 \mathrm{kHz}$, were level-equalised to $-26 \mathrm{dBov}$ and then bandpass-filtered complying with ITU-T Recommendation P.341. The response frequency of this filter is flat in the range 50 7,000 Hz, approximately. Next, the G.722 encoding and decoding processes were simulated by using standard ITU tools. After applying the codec, the signals were again level-equalised to $-26 \mathrm{dBov}$.

\section{Human Speaker Recognition from Logatomes}

A listening test was conducted to evaluate the human speaker recognition performance from speech of different phonetic content in different bandwidths. The goals were to ascertain which phonemes are effective for speaker recognition in NB and in $\mathrm{WB}$, to determine the improvement in performance gained when moving to the enhanced bandwidth for each phoneme, and to relate the obtained results to human speech intelligibility rates with the same speech material (Section 4).

Because it was assumed that the potential listeners in the listening test do not know the voices of the speakers of the OLLO database (i.e. they were unfamiliar), a human speaker verification scheme was adopted in this experiment. Two stimuli were presented to the listener, who was then asked to decide whether they corresponded to the same person or not. The logatome and the speaker gender in both stimuli were the same, varying only among verification trials. Different speakers tended to utter the logatomes with slightly different prosody. To avoid listeners' decisions guided by the same or the different prosody of the two stimuli rather than by relevant speaker-specific information, stimuli with different intonation were always presented. The first stimulus of each trial was uttered in normal speaking style and the second in question speaking style.

In the listening test, stimuli in the verification trials were either NB-NB (no mismatch, narrowband), WB-WB (no mismatch, wideband), or WB-NB (bandwidth mismatch). It can be expected that detecting the same speaker in two segments is more difficult for the WB-NB trials, as fewer speaker-specific characteristics may be preserved under bandwidth mismatch compared to the no mismatch situations. The same number of true trials (same speaker in both stimuli) and of false trials (different speakers in each stimulus) with balance in speaker gender were presented in different random order to each listener. In all, 288 trials were included in each test, resulting from twelve logatomes, three conditions, and eight trials (four true and four false).

30 listeners (16 males and 14 females) with German as their mother tongue took part in the listening test. Their age ranged from 20 to 35 years, with a mean of 27.0 years. Each of them participated in the listening test session individually. A simple program for presenting the trials and for logging the listeners' answers was written in Java. The listeners were instructed to click on a first and then on a second loudspeaker button to listen to each stimulus, and then on an equal button if they decided that the voices heard corresponded to the same person or on an unequal button otherwise. No time constraints were imposed, yet they could listen to each stimulus only once.

Each test session took 35 min approximately, including three breaks to avoid fatigue and loss of focus. The sessions were held in a quiet room and the test administered with a computer with a high-quality soundcard and Shure SRH240 headphones (frequency range $20-20,000 \mathrm{~Hz}$ ) with diotic listening. 


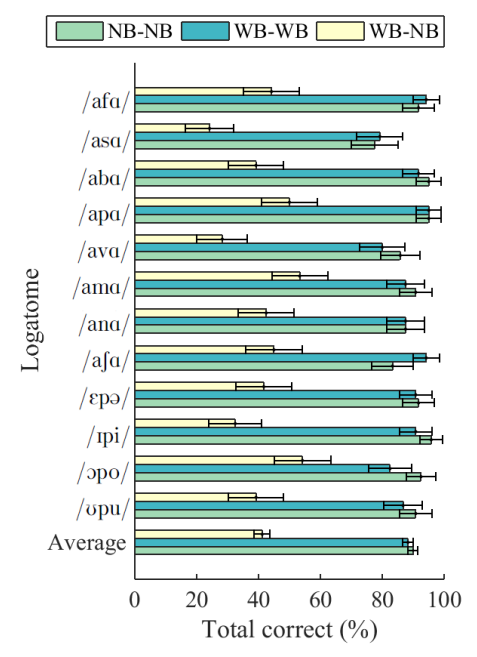

Figure 1: Human speaker recognition performance and $95 \%$ confidence intervals for each logatome considering only true trials. No statistical differences between $N B-N B$ and $W B-W B$ stimuli have been found.

The rates of correct acceptances (correct responses when the speaker was the same) and of correct rejections (correct responses when the speakers were different) are shown in Figures 1 and 2, respectively. The McNemar's statistical test was conducted to evaluate the significance of the differences between NB-NB and WB-WB accuracies. It can be observed that NB-NB stimuli generally led to high rates of correct acceptances but to low rates of correct rejections, which indicates that most of the voices sound similar to the listeners in NB, that is, the speaker-related properties of the voices are less noticeable. The WB-NB stimuli permitted high correct rejections and low correct acceptances, revealing that most of the voices, also those in same-speaker trials, were found to be different by the participants, which was caused by the bandwidth mismatch. The correct rejection rates with WB-WB stimuli were lower than with WB-NB stimuli except for the logatome /ama/.

The correct acceptance rates with WB-WB were generally lower than with NB-NB except for the logatomes /afa/, /asa/, and $/ \mathrm{a} \mathrm{a} \mathrm{a} /$. This fact evinces that the information conveyed by the unvoiced fricatives $/ \mathrm{f} /, / \mathrm{s} /$, and $/ \mathrm{f} /$ to determine whether two speakers are the same is enhanced when switching from NB to $\mathrm{WB}$ to a greater extent than for the other phonemes tested.

The differences between the NB-NB and WB-WB accuracies were better manifested in the case of false trials, where it was easier for the listeners to detect the differences between two voices with WB-WB than with NB-NB trials. The higher correct rejections permitted in WB compared to NB were significant $(p<0.001)$ for $/ \mathrm{afa} /, / \mathrm{ava} /$, and $/ \mathrm{ama} /$. The stop sounds in /aba/, /rpi/, /opo/ also offered significantly better performance in WB compared to NB, yet their accuracy in WB was statistically the same as chance level $(50 \%)$. A low rate of correct rejections reflects that the tested logatome contains speaker properties shared by more speakers, i.e. it is not useful to distinguish between speakers. The correct rejections rates in NB fell significantly below chance level for /aba/, /apa/,

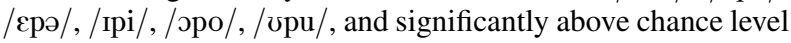
for /ava/ and /ama/ in WB.

The nasals in /ama/ and /ana/ offered among the high-

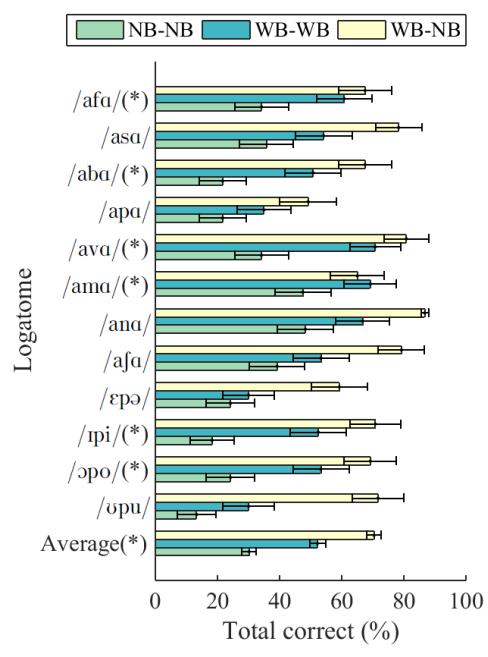

Figure 2: Human speaker recognition performance and $95 \%$ confidence intervals for each logatome considering only false trials. Significant statistical differences between $N B-N B$ and $W B-W B$ stimuli with $p<0.001$ are indicated with $\left({ }^{*}\right)$ for the corresponding logatomes.

est correct rejection rates in $\mathrm{NB}$ and in WB. This confirms the importance of nasal phonemes for speaker recognition as also assessed in [9]. Besides, the bandwidth extension caused significant difference in accuracies for the fricatives $/ \mathrm{afa} /$ and /ava/, which were not as effective as nasals in NB. Nasals and fricatives are known to possess speaker-specific properties, attributable to their articulatory and acoustic properties and relatively fixed nasal and paranasal cavities of the talkers [24, 11]. Nevertheless, while these properties are more distributed in the spectrum in the case of nasals, effective for speaker recognition in both bandwidths, the speaker-specific information carried by fricatives is concentrated on the higher frequencies. Hence, the transmission of fricatives through an extended bandwidth (WB) implies a significant improvement over NB in correct rejection rates, to a greater extent than for nasals. Stop sounds resulted to be less useful for speaker discrimination, in concordance with other results in the literature $[9,6]$.

\section{Intelligibility from Logatomes}

A speech intelligibility test was conducted with the same speech material described in Section 2 to find differences between NB and WB in the detection of particular phonemes, to quantify the possible improvement of intelligibility with WB communications over NB, and to relate this to the human speaker recognition results of Section 3 .

The total of stimuli heard by each listener was 192, resulting from twelve logatomes, two channel conditions, and eight repetitions from different speakers (randomly selected from the OLLO set of ten speakers with gender balance). The detection of consonants by humans is a relatively easy task in the absence of noise. In order to avoid accuracies close to $100 \%$, which would not show differences among the phonemes or between the bandwidths, the question speaking style was selected for the logatomes. This speaking style may offer intelligibility rates somewhat lower than those for normal style [16].

The same group of 30 listeners of the speaker verifica- 


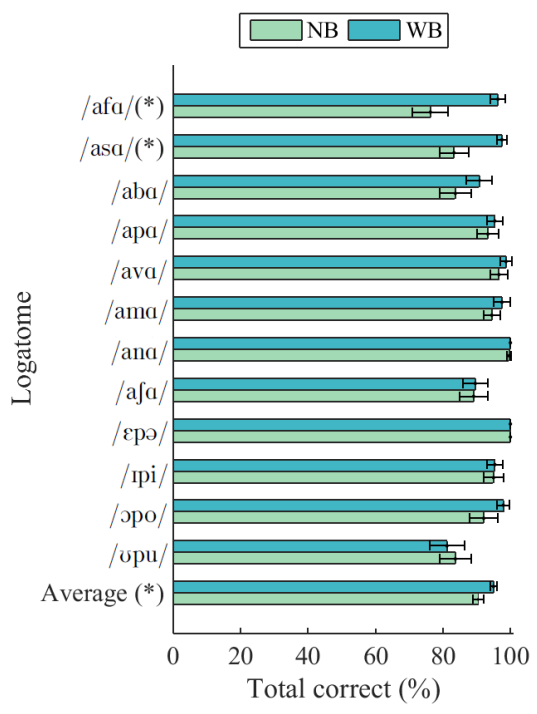

Figure 3: Accuracies and 95\% confidence intervals detecting logatomes in NB and in WB. Significant statistical differences between NB and WB stimuli with $p<0.001$ are indicated with (*) for the corresponding logatomes.

tion test participated in the intelligibility test, on the same day, and employing the same computer and headphones in the same room. Their task was to choose a logatome by clicking on one item from a list of logatomes after listening to each transmitted stimulus. The different options were presented on the computer screen. The test took about 12 min to complete, including one break.

The average accuracy reached by the group of listeners was $92.80 \%$ with a standard deviation of $25.85 \%$. Figure 3 presents the accuracies in NB and in WB for each logatome. Despite the high and almost saturated accuracy, caused by the relatively non-severe distortions of the stimuli, significant differences between the two bandwidths could be obtained. The McNemar's test indicated that the difference between the NB and the WB accuracies is statistically significant $(p<0.001)$ for $/ \mathrm{afa} /$, for /asa/, and considering all logatomes pooled. The intelligibility accuracy was improved from NB to WB in every case, except for the logatome / vpu/. 22 out of 30 listeners reported they had had difficulties distinguishing between /opo/ and / vpu/ because of the ambiguous realisations of some speakers, which were perceived as /opu/.

The work in [17], employing CVC logatomes, showed the recognition accuracy of different consonants employing highpass filters with increasing cut-off frequencies. The accuracy detecting /s/ and /f/ decreased only with a cut-off frequency above $8 \mathrm{kHz}$ and $10 \mathrm{kHz}$, respectively. Other fricatives and affricates such as $/ \theta, \int, \mathrm{t} \int, \mathrm{d}_{3} /$, with frication energy concentrated in lower frequencies, offered an earlier drop in detection performance, when they were high-pass filtered at $4 \mathrm{kHz}$. Stop sounds such as $/ \mathrm{p} /$ and $/ \mathrm{b} /$ were less affected by the high-pass filtering. In the experiments of this section, the relevance of the higher frequencies for the recognition of $/ \mathrm{f} /$ and $/ \mathrm{s} /$, not manifested for other phonemes tested, is confirmed. The inclusion of the high frequencies $3.4-7 \mathrm{kHz}$ in the speech bandwidth enables a significantly better discrimination between /f/ and /s/. The range $50-300 \mathrm{~Hz}$ is also included in WB with respect to
$\mathrm{NB}$, although this low frequency range presumably offers little benefit in comparison to the high frequencies.

The greatest confusion between logatomes was found in NB between $/ \mathrm{afa} /$ and /asa/ reciprocally, /s/ being better detected than $/ \mathrm{f} /$. When switching to the enhanced bandwidth, the total number of errors with each of these logatomes was reduced from 82 in NB to 13 in WB, out of 240 logatome presentations in each bandwidth. This error reduction is of approximately factor 6 . The decrease of confusions was hypothesised, since /s/ and /f/ have similar spectral characteristics in NB but different in WB. Most of the spectral energy of / $/ \mathrm{s}$ is concentrated in the higher frequency range incorporated by WB whereas the energy in the /f/ spectrum is more uniformly distributed. A more detailed analysis of the intelligibility results can be found in [25].

\section{Relation Between Speaker Recognition and Intelligibility in NB and in WB}

The main purpose of this study was to investigate the possible relations between intelligible phonemes and phonemes enabling better speaker recognition in the transition from NB to WB. By employing logatomes as speech material and simulated telephone channel transmissions, the attention is focused on the effects of different phonemes rather than on overall word intelligibility rates. Both human speaker recognition and speech intelligibility were improved when moving from NB to WB. Our results indicate that the fricative /f/, significantly better detected in WB speech compared to NB $(p<0.001)$, also permits significantly better speaker recognition performance in the extended bandwidth $(p<0.001)$.

A weaker relation between speaker recognition and intelligibility scores was found for the rest of logatomes. Other phonemes also contributed to improved speaker recognition in WB: the nasals $/ \mathrm{m} /$ and $/ \mathrm{n} /$, and the fricative $/ \mathrm{v} /$, although for these phonemes no significant differences in speech intelligibility were found between the two bandwidths.

\section{Conclusions}

Two listening tests have been performed employing VCV logatomes previously transmitted through NB and WB channels. The first test examined the human speaker recognition performance in terms of correct acceptances and correct rejections, and the second one the intelligibility accuracy. Our main conclusion is that the speaker-discriminative potential of some fricative sounds is manifested in WB, where /f/ and /s/ are more intelligible.

The fricative sounds in /afa/, /a a a/, and /ava/, with energy concentrated in the upper part of the spectrum, carry important speaker-specific information that allows higher human speaker verification performance in WB compared to NB. The differences in performance between both bandwidths are greater than those caused by other phonemes such as the nasal sounds $/ \mathrm{m} /$ and $/ \mathrm{n} /$, which also convey speaker properties yet are more widely distributed in the spectrum. Regarding human speech intelligibility, the logatomes /afa/ and /asa/ are significantly more intelligible in WB than in NB. /afa/ is six times more confusable with /asa/ in NB compared to WB, due to the similarities of the NB spectra of the fricatives /f/ and /s/.

Future work will examine phoneme intelligibility by performing other tests, such as the CLID test. The effect of various transmission conditions will be investigated, incorporating super-wideband channels $(50-14,000 \mathrm{~Hz})$, which are currently preferred for HD video-conferencing applications. 


\section{References}

[1] J. Kreiman, B. R. Gerratt, K. Precoda, , and G. S. Berke, "Individual Differences in Voice Quality Perception," Journal of Speech and Hearing Research, vol. 35, no. 3, pp. 512-520, 1992.

[2] O. Baumann and P. Belin, "Perceptual Scaling of Voice Identity: Common Dimensions for Different Vowels and Speakers," Psychological Research, vol. 74, pp. 110-120, 2010.

[3] R. E. Remez, J. M. Fellowes, and P. E. Rubin, "Talker Identification Based on Phonetic Information," Journal of Experimental Psychology: Human Perception and Performance, vol. 23, no. 3, pp. 651-666, 1997.

[4] J. J. Wolf, "Efficient Acoustic Parameters for Speaker Recognition," The Journal of the Acoustical Society of America, vol. 51, no. 6 (Part 2), pp. 2044-2056, 1972.

[5] M. R. Sambur, "Selection of Acoustic Features for Speaker Identification," IEEE Transactions on Acoustics, Speech, and Signal Processing, vol. 23, no. 2, pp. 176-182, 1975.

[6] J. P. Eatock and J. S. Mason, "A Quantitative Assessment of the Relative Speaker Discriminating Properties of Phonemes," in IEEE International Conference on Acoustics, Speech, and Signal Processing (ICASSP), 1994, pp. 1133-1136.

[7] K. Stevens, C. Williams, J. Carbonell, and B. Woods, "Speaker Authentication and Identification: A Comparison of Spectrographic and Auditory Presentations of Speech Material," The Journal of the Acoustical Society of America, vol. 44, no. 6, pp. 1596-1607, 1968

[8] K. Amino and T. Arai, "Contribution of Consonants and Vowels to the Perception of Speaker Identity," in Japan-China Joint Conference of Acoustics, 2007.

[9] K. Amino, T. Sugawara, and A. Takayuki, "The Correspondences Between the Perception of the Speaker Individualities Contained in Speech Sounds and Their Acoustic Properties," in Annual Conference of the International Speech Communication Association (Interspeech), 2005, pp. 2025-2028.

[10] K. Amino, T. Osanai, T. Kamada, H. Makinae, and T. Arai, "Effects of the Phonological Contents and Transmission Channels on Forensic Speaker Recognition," in Forensic Speaker Recognition: Law Enforcement and Counter-Terrorism, A. Neustein and H. A. Patil, Eds. Berlin, Germany: Springer, 2011, pp. 275-308.

[11] K. N. Stevens, Acoustic Phonetics. Cambridge, UK: MIT Press, 1998.

[12] S. C. Creel and M. R. Bregman, "How Talker Identity Relates to Language Processing," Language and Linguistics Compass, vol. 5, no. 5, pp. 190-204, 2011.

[13] R. van Bezooijen and V. van Heuven, "Assessment of Synthesis Systems," in Handbook of Standards and Resources for Spoke Language Systems, D. Gibbon, R. Moore, and R. Winski, Eds. New York, NY, USA: Walter de Gruyter, 1997, pp. 481-563.

[14] G. A. Miller and P. E. Nicely, "An Analysis of Perceptual Confusions Among Some English Consonants," The Journal of the Acoustical Society of America, vol. 27, no. 2, pp. 338-352, 1955.

[15] S. A. Phatak, A. Lovitt, and J. B. Allen, "Consonant Confusions in White Noise," The Journal of the Acoustical Society of America, vol. 124, no. 2, pp. 1220-1233, 2008.

[16] B. T. Meyer, T. Jürgens, T. Wesker, T. Brand, and B. Kollmeier, "Human Phoneme Recognition as a Function of Speech-Intrinsic Variabilities," The Journal of the Acoustical Society of America, vol. 128, no. 5, pp. 3126-3141, 2010.

[17] R. P. Lippmann, "Accurate Consonant Perception Without MidFrequency Speech Energy," IEEE Transactions on Speech and Audio Processing, vol. 4, no. 1, pp. 66-69, 1996.

[18] E. W. Healy, S. E. Yoho, and F. Apoux, "Band Importance for Sentences and Words Reexamined," The Journal of the Acoustical Society of America, vol. 133, no. 1, pp. 463-473, 2013.
[19] M. Balestri, E. Foti, L. Nebbia, M. Oreglia, P. L. Salza, and S. Sandri, "Comparison of Natural and Synthetic Speech Intelligibility for a Reverse Telephone Directory Service," in International Conference on Spoken Language Processing (ICSLP), vol. 1, 1992, pp. 559-562.

[20] S. Möller, A. Raake, N. Kitawaki, A. Takahashi, and M. Wältermann, "Impairment Factor Framework for Wideband Speech Codecs," IEEE Transactions on Audio, Speech, and Language Processing, vol. 14, no. 6, pp. 1969-1976, 2006.

[21] L. Fernández Gallardo, S. Möller, and M. Wagner, "Human Speaker Identification of Known Voices Transmitted Through Different User Interfaces and Transmission Channels," in IEEE International Conference on Acoustics, Speech, and Signal Processing (ICASSP), 2013, pp. 7775-7779.

[22] J. Rodman, "The Effect of Bandwidth on Speech Intelligibility," 2003, polycom, White Paper.

[23] S. E. Stuntz, "Speech-Intelligibility and Talker-Recognition Tests of Air Force Voice Communications Systems," Electronic System Division, Air Force Systems Command, U.S. Air Force, Tech. Rep. ESD-TDR-63-224, 1963.

[24] M. Gordon, P. Barthmaier, and K. Sands, "A Cross-Linguistic Acoustic Study of Voiceless Fricatives," Journal of the International Phonetic Association, vol. 32, no. 2, pp. 141-174, 2002.

[25] L. Fernández Gallardo and S. Möller, "Phoneme Intelligibility in Narrowband and in Wideband Channels," in Annual German Congress on Acoustics (DAGA), 2015. 